\title{
Effect Of Preoperative Nutrition Modulation On Postoperative Complications And Short -Term Outcome In Major Abdominal Cancer Surgery : An Immunological View
}

\section{1- Prof . Dr Osama Aly Mohammed Ibraheim}

Professor of anaeshtesia, ICU, and pain mangement faculty of medicine, Assiut University.

E-mail : osamaibrahiem@gmail.com

Telephone number: 01069009803

\section{2- Prof .Dr Khaled Mohamed Fares}

Professor of anaeshtesia, ICU, and pain mangement, South Egypt Cancer Istitute.

E-mail: faressali@yahoo.com

Telephone number: 01283757288

\section{3- Dr . Montaser Abdelfataah Mohamed}

Assistant Professor of anaeshtesia, ICU, and pain mangement, South Egypt Cancer Istitute

E-mail: Abdelfatahmontaser69@yahoo.com

Telephone number: 01090939346

\section{4- Romany Gergis Naeem Gendy}

Demonstrator of Anaesthesia and I.C.U ,South Egypt Cancer Istitute, Assiut University

E-mail: romanytreka22@gmail.com

Telephone number: 01006231332

published at 19/6/2018 


\section{Abstract:}

Background: There has been a positive correlation between malnutrition and increased risk of infections. Additionally, protein caloric malnutrition occurs in up to $90 \%$ of surgical oncology patients . Malnutrition along with surgical stress predisposes patients to significant postoperative complications and immune depressions. Therefore, strategies have been implemented to circumvent malnutrition such as avoiding preoperative fasting, re-establishing oral feeding as soon as possible and maintaining good glycemic and metabolic control.

Objective: We hypothesised that perioperative use of various biochemical agents such as non-essential ( glutamine, arginine ) or sulfur-containing amino acids, omega -3-poly unsaturated fatty acids, nucleotides and antioxidants ( free radical scavengers ), may alter the immune response and could improve postoperative outcome in gastrointestinal cancer patients undergoing surgeries.

Patients \& Methods: This study was done after obtaining approval of the local ethics committee of faculty of medicine, Assiut University This randomized double -blinded prospective study was conducted on thirty patients. The patients were divided into two groups : Group1: $(n=15)$ patients received preoperative I.V solutions as a control group and also received total parenteral nutrition immediately postoperative for 5 day, Group $2:(n=15)$ patients received 5 days preoperative (SMOF lipid ) $500 \mathrm{ml} /$ day in a peripheral venous line and also recieved total parenteral nutrition immediately postoperative in a central venous line as group (1) for 5 days. The effect of immunonutrition on the two groups was evaluated according to the WBC level , and the incidence of postoperative complications ( surgical site infection, fistula, chest infection ), and hospital length of stay .

Results: There were non significancant difference between the study groups inWBC level in the prenutritional, preoperative, postoperative day 1 , but there were a significant difference in the post operative day 3 samples, and in the post operative day 5 samples. There were significant difference between the two groups in the incidence of surgical site infection, and hospital stay postoperative. There were non significant difference between the two groups in the incidence of the fistula and chest infection postoperative.

Conclusion: Preoperative immunonutrition support is effective in reducing postoperative complications in cancer patients. It helps to lower the risk of postoperative infectious complications and hospital lengh of stay.

Key Words: Preoperative immunonutrition, cancer patients, total parenteral nutrition. 


\section{Introduction:}

Malnutrition is a common finding in patients presenting for surgical management of abdominal malignancies, with an estimated prevalence in this group of $40 \%$ to $80 \%{ }^{(1)}$. A complex mix of factors, such as tumor location, tumor type, stage of disease, and preoperative radiation and / or chemotherapy treatments ,might predispose patients to malnutrition . Nausea, vomiting, reduced appetite, early satiety, taste changes, diarrhea , pain , mucositis , physical obstruction and malabsorption could result in weight loss, which in turn is a strong prognostic indicator of poor outcome in terms of survival and response to treatment $(\mathbf{1})$. The further catabolic stress of major surgery increases the risk of cancer related malnutrition, which correlates with increased rates of perioperative morbidity and mortality (2)

Patients who are malnourished are more likely to suffer from higher rates of morbidity and mortality as well as longer hospital stays ${ }^{(\mathbf{3})}$

Several studies have attempted to identify optimal nutrition strategies in such patients . It is widely accepted practice that patients undergoing elective surgery with $>10 \%$ weight loss or expected fasting more than week postoperatively should receive artificial nutrition ${ }^{(4)}$

Postoperative nutrition supplementation has been shown to have a beneficial effect on surgical outcomes ${ }^{(5)}$.

Reasons purported for this practice include reducing the risk of postoperative abdominal distension, nausea, vomiting, and subsequent concerns regarding anastomotic breakdown, wound dehiscence and pulmonary aspiration ( 6).

Malnutrition can suppress immune function , reduce host defense and increase incidence of infection (7). Malnutrition decrease both cell mediated and humeral immune responses, which are restorted with re-feeding ${ }^{(8)}$. In fact, immune abnormalities in the majority with malnutrition could be reversed after adequate nutrition $^{(9)}$

\section{The present study aimed to :}

A) Detect the effect of preoperative immunonutrition on CBC 5: days preoperative (prenutritional sample) , before the operation ( immediately preoperative ), on postoperative day 1 (POD1), POD3, and POD5.

B) Postoperative infections: surgical site infection, intra-abdominal or pelvic infection or abscess, anastomotic leakage (fistula) in the prenutritional, preoperative, post operative day 1 , POD3, and POD5.

\section{Patients and methods:}

After obtaining an approval from the local ethics committee of faculty of medicine, Assiut University , Assiut , Egypt , and patients' written informed consent, this randomized double -blinded prospective study was conducted on thirty patients aged from 18 to 70 years with primary abdominal cancer, and of American Society of Anesthesiologist (ASA) physical status III or IV scheduled for major abdominal cancer surgeries.

Patients older than 70 years and younger than 18 years, metastatic cancer or

According to a computer- generated randomized number table, patients were assigned to two groups of 15 patients each :

Group 1: patients recieved preoperative I.V solutions as a control group and also received total parenteral nutrition(TPN) immediately postoperative for 5 days, the TPN regimen consisted of carbohydrate( glucose 25\%), protein ( Amino sterile), trace elements and glutamine and fat (SMOF lipid ) containing soya bean oil, medium chain triglyceride , olive oil and fish oil rich in omega-3- fatty acids in a central venous catheter which will be insereted intraoperatively.

Group2: patients received 5 days preoperative (SMOF lipid ) $500 \mathrm{ml} /$ day in a peripheral venous line and also recieved total parenteral nutrition immediately postoperative in a central venous line as group (1) for 5 days .

Postoperative caloric requirements were calculated by Harris-Benidict Equation .This equation is used to predict the basal energy expenditure (BEE) which is the basal metabolic rate in the resting and fasting states in a thermo-neutral environment, which is corrected by considering both activity and stress factors. This equation depends on the age, sex, height , and ideal body weight.

-Male's BEE (kcal/day) $=66.5+(13.75 \times$ body weight in $\mathrm{Kg})+(5.003 \times$ height in $\mathrm{cm})-($ $6.775 \times$ age in years) . 
-Female's BEE (kcal/day) $=655.1+(9.563 \times$ body weight in $\mathrm{Kg})+(1.850 \times$ height in $\mathrm{cm})-$ ( 4.676x age in years ).

-TEE ( Total Energy Expenditure ) (kcal/day) $=$ BEE $\times$ Stress factor $\times$ Activity factor.

-Stress factors : (Surgery and infection 1.2), (Trauma 1.5 ), ( Sepsis 1.6),

(Burns 1.6-2).

-Activity factors : ( sedentery 1.2), ( Normal activity 1.3 ), (Active 1.4). (Very active 1.5) -Protein requirements per day : in the range of $1.5-2.5 \mathrm{~g} / \mathrm{kg}$.

(Post-operative, cancer, inflammatory $1.5 \mathrm{~g} / \mathrm{kg}$ ), ( Sepsis , Polytrauma 2 g/kg), (Peritonitis, burns $2.5 \mathrm{~g} / \mathrm{kg}$ ).

-The patients' immune response assemment was according to the level of WBCs, the rate of post operative infection( surgical site infection, fistula , and chest infection ), and the hospital length of stay .

All the patients were provided routine perioperative care with antibiotic prophylaxis, multimodal analgesia , deep venous thrombosis prophylaxis and early mobilization. recurrent, or with organ failure ( liver or renal insufficiency ), presence of ongoing infections , patients suffered from inflammatory bowel diseases, and patients with history of recent immunosuppressive or immunologic disease, will be excluded from the study.

\section{Statistical analysis:}

Data entry and analysis were done using SPSS version 20 ®(Statistical Package for Social Science). Data were presented as number, percentage, mean \pm standard deviation. Chi-square test was used to compare between qualitative variables. Mann-Whitney test was used to compare quantitative variables between the studied groups. Wilcoxon Signed Rank test was done to compare between each two times in the same group. P-value considered statistically significant when $\mathrm{P}<$ 0.05. Sample size was calculated using EPI info program 2000. At power $80 \%$ and confidence interval 95\%, 15 patients were required in each group.

\section{Discussion:}

The main cause of cancer death is tumor recurrence and metastasis that occur even after removal of a primary tumor by surgery . Cancer patients are immunosuppressed as a result of their own disease, chemotherapy, radiation therapy, malnutrition, and psychological stress, which all induce apoptosis of immune cells. Therefore, cancer patient's show depressed immune functions (10).
Historically, there has been a positive correlation between malnutrition and increased risk of infections. Additionally, protein caloric malnutrition occurs in up to $90 \%$ of surgical oncology patients. Malnutrition along with surgical stress predisposes patients to significant postoperative complications and immune depression ${ }^{(\mathbf{1 1}, \mathbf{1 2}) \text {. }}$

Therefore, strategies have been implemented to circumvent malnutrition such as avoiding preoperative fasting, re-establishing oral feeding as soon as possible and maintaining good glycemic and metabolic control. Furthermore, research is now demonstrating the importance of additional perioperative nutritional support through the use of enteral and parenteral feeds in high-risk patients

The immunonutrients are glutamine, arginine, and polyunsaturated fatty acids (omega-3), among others, which can improve the immunity and nutrition effectively. They can modulate inflammatory responses and enhance protein synthesis and then increase immune responses. It has been proved that the perioperative application of immunonutrition is an effective and more prevailing therapeutic strategy (13).

In this study there were significant difference $(\mathrm{P}<0.05)$ between the two studied groups in the incidence of surgical site infection and length of hospital stay. This agree with Rowan and his collaborators who concluded that perioperative immunonutrition may lead to significant reductions of postoperative complications. Silvestri et al. compared the clinical characteristics of patients after pancreaticoduodenectomy between immunonutrition group and control group and found that immunonutrition helps to lower the risk of postoperative infectious complications (14)

Although some studies showed no significant difference with hospital stay in immunonutrition group, different outcome was observed in our study. We attribute these results to the choice of the subjects. Moya and his team studied the effect of immunonutrition on the patients under colorectal resection in an enhanced recovery after surgery (ERAS) protocol and drew a conclusion that the average postoperative hospital stay was not significantly different between the two groups. Their median postoperative hospital stay, which was only 5 days, maybe interfered by the applications of ERAS ${ }^{(\mathbf{1 5}) .}$ 


\section{Conclusion:}

Preoperative immunonutrition support is effective in reducing postoperative complications in cancer patients. It helps to lower the risk of postoperative infectious complications and hospital lenght of stay.

\section{References:}

1. Lighvani, DM, Frucht D, Jankovic et al., "T-bet is rapidly induced by interferon$\gamma$ in lymphoid and myeloid cells," Proceedings of the National Academy of Sciences of theUnited States of America, vol. 98, no. 26, pp. 15137-15142, 2001.

2. Mullen C, Hutchins AS, High FA. et al., "Hlx is induced by and genetically interacts with T-bet to promote heritable THI gene induction," Nature Immunology, vol. 3, no. 7, pp.652-658, 2002.

3. Iwasaki and, Medzhitov R, “Toll-like receptor control of the adaptive immune responses," Nature Immunology, vol. 5, no. 10, pp. 987-995, 2004.

4. Abendstein H, Nordgren M, Boysen M, et al. Quality of life and head and neck cancer: a 5-year prospective study. Laryngoscope 2005; 115(12):2183-92.

Ates E, Yilmaz S, Erkasap S, Ihtiyar E, Kaya Y, Pehlivan $T$, et al. Perioperative immunonutrition ameliorates the postoperative immune depression in patients with gastrointestinal system cancer (prospective clinical study in 42 patients). Acta Gastro-enterologica Belgica 2004;67(3):250e4

5. Oestreich KJ, Huang AC, and Weinmann AS., "The lineage-defining factors $\mathbf{T}$-bet and Bcl-6 collaborate to regulate Th1 gene expression patterns," Journal of Experimental Medicine, vol. 208, no. 5, pp. 1001-1013, 2011.

6. Bansal V, Ocha JB , Argininie availability, arginase and the immune response . Curr opin ClinNutr meteb care 2003;6:223-8.

7. Barbosa VM, Miles EA, Calhau C, et al. Effects of a fish oil containing lipid emulsion on plasma phospholipid fatty acids, inflammatory markers, and clinical outcomes in septic patients: a randomized, controlled clinical trial. Crit Care 2010;14(1):R5.

8. Hennessey D, Burke JP, Mealy K. Malnutrition and postoperative complications in abdominal surgery. Annals of Surgery 2011;254(4):666.
9. Kurosawa S. Anesthesia in patients with cancer disorders. Curr Opin Anaesthesiol 2015;25:376-84.

10. Garth AK, Newsome CM, Simmance N, et al. Nutritional status, nutrition practices and post-operative complications in patients with gastrointestinal cancer. J Hum Nutr Diet 2010;23:393-401.

11. Bozzetti F, Gianotti L, Braga M, et al. Postoperative complications in gastrointestinal cancer patients: the joint role of the nutritional status and the nutritional support. Clin Nutr 2007;26:698-709.

12. Popovic PJ, Zeh HJ, Ochoa JB. Arginine and immunity. J Nutr 2007;137:S1681-6.

13. Rowan NR, Johnson JT , Fratangelo CE, Smith BK, Kemerer PA, Ferris R. L.

Utility of a perioperative nutritional intervention on postoperative outcomes in high-risk head \& neck cancer patients. Oral Oncology. 2016;54:42-46. doi: 10.1016/j.oraloncology.2016.01.006.

14. Moya P, Soriano-Irigaray L, Ramirez JM , et al. Perioperative standard oral nutrition supplements versus immunonutrition in patients undergoing colorectal resection in an enhanced recovery (ERAS) protocol. Medicine. 2016;95(21).

15. Yanzhong Xin, 1 Hongfei Cai, 1 LihuiWu,2 and Youbin Cuil, The Effect of Immunonutrition on the Postoperative Complications in Thymoma with Myasthenia Gravis. Hindawi Publishing Corporation Mediators of Inflammation Volume 2016, Article ID 8781740, 8 pages.

16. Calder PC. Fatty acids and inflammation from the membrane to thenucleus and from the laboratory bench to the clinic. Clinical Nutrition. 2010;29(1):5-12. 10.1016/j.clnu.2009.11.003.

In the present study were non significance difference in WBC $(\mathrm{P}>0.05)$ between two groups .At pre-nutritional, preoperative, at day1 and day3. But there were significance difference $(\mathrm{P}<0.05)$ at day5 with lower values in IN group. This agree with Yanzhong Xin et al., ${ }^{(18)}$ reported that the inflammatory markers including WBC count were decreased in the IN group of patients with severe intracranial hemorrhage during the acute stage ${ }^{(16) .}$ 
Calder reported that omega $3(\omega-3)$ polyunsaturated fatty acids ( $\omega$ - 3 PUFAs), as a main component of the immune nutrients, ensured the maintenance of membrane fluidity and the sufficient function of membrane proteins. Its dysbolism maybe leads to many human diseases including immune disorders or cancers (17).

Table (1): Patients demographic and clinical data in the two studied groups :

\begin{tabular}{|c|c|c|c|}
\hline Item & G1"n=15" & G2"n=15" & p-value \\
\hline $\begin{array}{ll}\text { 1- } & \text { Age } \\
\text { 2- } & \text { Sex: } \\
& \bullet \quad \text { Male } \\
& \bullet \quad \text { female } \\
\text { 3- } & \text { Weight } \\
\text { 4- } & \text { Height } \\
\text { 5- } & \text { BMI } \\
\text { 6- } & \text { Operation time "hours" } \\
\text { 7- } & \text { Blood loss "ml" }\end{array}$ & $\begin{array}{c}55.26 \pm 6.89 \\
7(23.3 \%) \\
8(53.3 \%) \\
70.60 \pm 6.73 \\
166.07 \pm 6.43 \\
25.59 \pm 1.89 \\
4.06 \pm 0.66 \\
560.00 \pm 80.62\end{array}$ & $\begin{array}{c}7(23.3 \%) \\
8(53.3 \%) \\
69.46 \pm 9.17 \\
166.8 \pm 7.26 \\
24.85 \pm 1.46 \\
3.96 \pm 0.76 \\
556.67 \pm 0.97\end{array}$ & $\begin{array}{l}\mathrm{P}=0.703 \mathrm{n} . \mathrm{s} \\
\mathrm{P}=0.772 \mathrm{n} . \mathrm{s} \\
\mathrm{P}=0.242 \mathrm{n} . \mathrm{s} \\
\mathrm{P}=0.701 \mathrm{n} . \mathrm{s} \\
\mathrm{P}=0.920 \mathrm{n} . \mathrm{s}\end{array}$ \\
\hline
\end{tabular}

Data are represented on mean $( \pm \mathrm{SD})$, number, and percentages. $\mathrm{P}<0.05$.

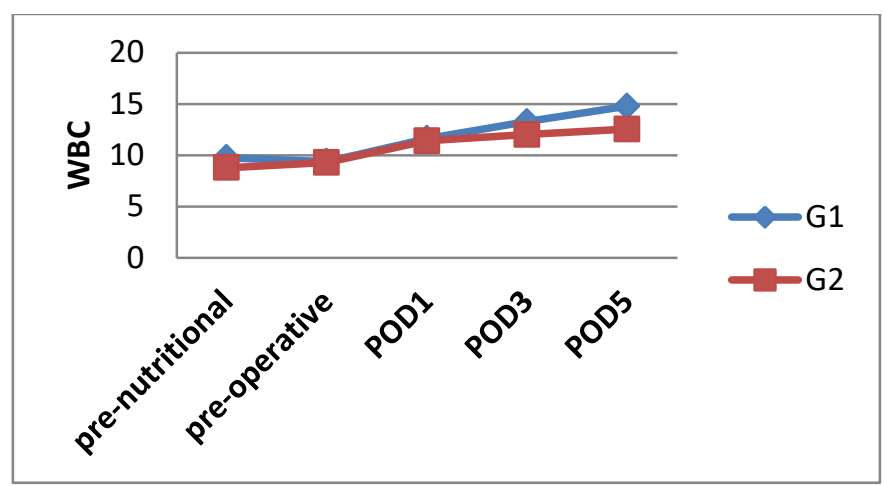

Fig (1): Changes in the mean WBC count with time in the two studied groups 


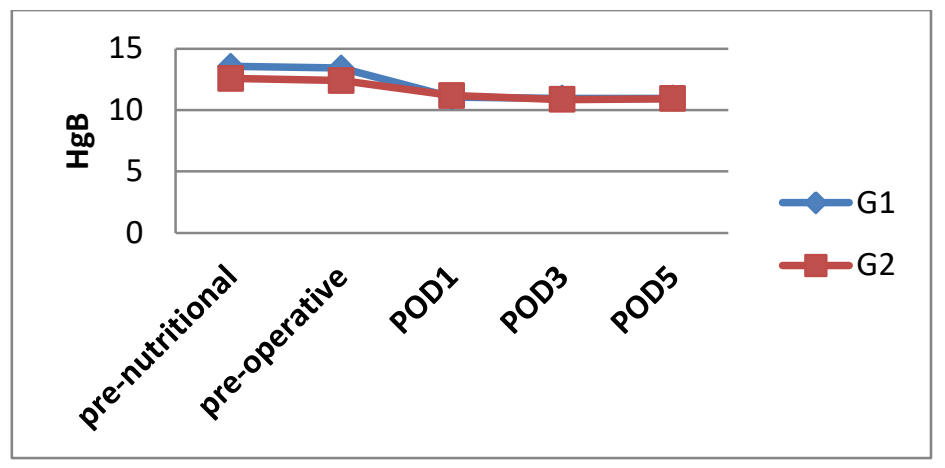

Fig (2): Changes in the mean $\mathrm{Hg}$ level with time in the two studied groups

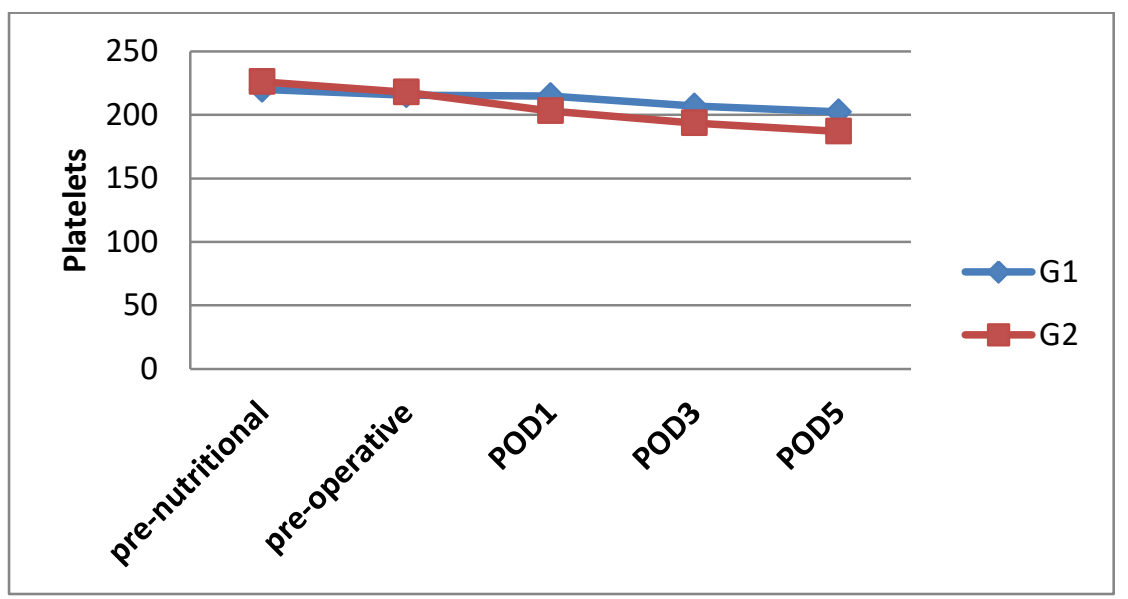

Fig (3): Changes in the mean Platlet count with time in the two studied groups.

Table (2): Postoperative side effects and hospital length of stay in the two studied groups :

\begin{tabular}{||l||c||c||c||}
\hline Item & G1"n=15” & G2"n=15” & p-value \\
\hline $\begin{array}{l}\text { 1- } \begin{array}{l}\text { Surgical site infection: } \\
\text { No }\end{array} \\
\text { 2-Fistula }\end{array}$ & $5(33.3 \%)$ & $2(13.3 \%)$ & P<0.01* \\
$\quad$ Yes & $10(66.7 \%)$ & $13(86.7 \%)$ & \\
$\quad$ No & $3(20.0 \%)$ & $1(6.7 \%)$ & P=0.299n.s \\
3-Chest infection: & $12(80.0 \%)$ & $14(93.3 \%)$ & \\
$\quad$ Yes & & & \\
$\quad$ No & $4(26.7 \%)$ & $2(13.3 \%)$ & P=0.326n.s \\
4-Hospital lengh of stay & $11(73.3 \%)$ & $13(86.7 \%)$ & P<0.02* \\
( days ) & $13.53 \pm 7.13$ & $10.93 \pm 4.23$ & \\
\hline \hline
\end{tabular}

